\title{
3D Crowd Counting via Multi-View Fusion with 3D Gaussian Kernels
}

\author{
Qi Zhang, Antoni B. Chan \\ Department of Computer Science, City University of Hong Kong \\ Tat Chee 83, Kowloon Tong, Hong Kong SAR, China \\ qzhang364-c@my.cityu.edu.hk, abchan@cityu.edu.hk
}

\begin{abstract}
Crowd counting has been studied for decades and a lot of works have achieved good performance, especially the DNNs-based density map estimation methods. Most existing crowd counting works focus on single-view counting, while few works have studied multi-view counting for large and wide scenes, where multiple cameras are used. Recently, an end-to-end multi-view crowd counting method called multiview multi-scale (MVMS) has been proposed, which fuses multiple camera views using a $\mathrm{CNN}$ to predict a $2 \mathrm{D}$ scenelevel density map on the ground-plane. Unlike MVMS, we propose to solve the multi-view crowd counting task through 3D feature fusion with 3D scene-level density maps, instead of the 2D ground-plane ones. Compared to 2D fusion, the $3 \mathrm{D}$ fusion extracts more information of the people along $z$ dimension (height), which helps to solve the scale variations across multiple views. The 3D density maps still preserve the 2D density maps property that the sum is the count, while also providing $3 \mathrm{D}$ information about the crowd density. We also explore the projection consistency among the 3D prediction and the ground-truth in the $2 \mathrm{D}$ views to further enhance the counting performance. The proposed method is tested on 3 multi-view counting datasets and achieves better or comparable counting performance to the state-of-the-art.
\end{abstract}

\section{Introduction}

Single-view crowd counting has been studied for decades and has achieved promising performance on the existing counting datasets (Zhang, $\mathrm{Li}$, and et al. 2015; Idrees et al. 2013; Zhang and et al. 2016; Chan, Liang, and Vasconcelos 2008; Chen et al. 2012; Idrees and et al. 2018). However, in real-world applications, there are several situations where single-view counting cannot perform well (e.g., see Fig. 1): 1) The scene is too wide (such as a park or a football match stadium) where a single camera's field-of-view is limited and cannot cover the whole scene; 2) The scene is too long (such as the underground train platform) where the people who are far away from the camera have very low resolution and the counting methods' performance drops on these people; 3) The scene contains a lot of obstacles, such as vehicles, building structures or etc., where many people are

Copyright (c) 2020, Association for the Advancement of Artificial Intelligence (www.aaai.org). All rights reserved.

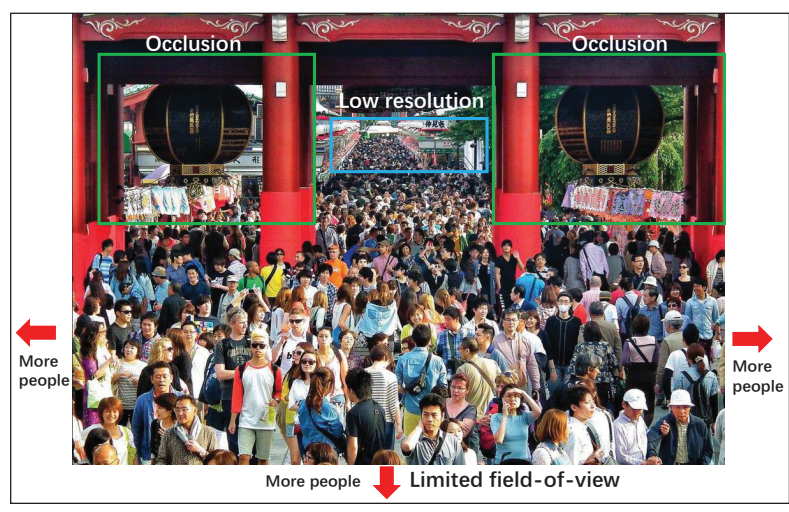

Figure 1: An example for the limitation of single-view counting for large and wide scenes: limited field-of-view, low resolution and severe occlusion. This image is from the ShanghaiTech dataset (Zhang, Li, and et al. 2015).

heavily or totally occluded. Under the 3 conditions, the current single-view counting methods are inaccurate, because many people are miscounted due to limited field-of-view, low resolution and severe occlusion.

To address the aforementioned situations, multiple cameras should be deployed, and the multi-view information should be fused to enhance the counting performance for complicated scenes. A few works (Li, Huang, and Liu 2012; Ryan et al. 2014; Tang et al. 2014; Ge and Collins 2010) have considered multi-view counting. However, the hand-crafted features and the foreground extraction steps limit their performance. Recently, a CNN-based multi-view counting method (Zhang and Chan 2019) was proposed, which improved the counting performance on wide-area scenes. In their paper, the multi-view information (cameraview density maps or feature maps) are projected to the same 2D plane in the 3D scene (at the height of a person), and then fused to predict the 2D scene-level density maps on the ground-plane. Several fusion methods are considered, including late and early fusion, and a multi-view multi-scale early fusion model (MVMS), which handles both the interand intra-view scale variations. 


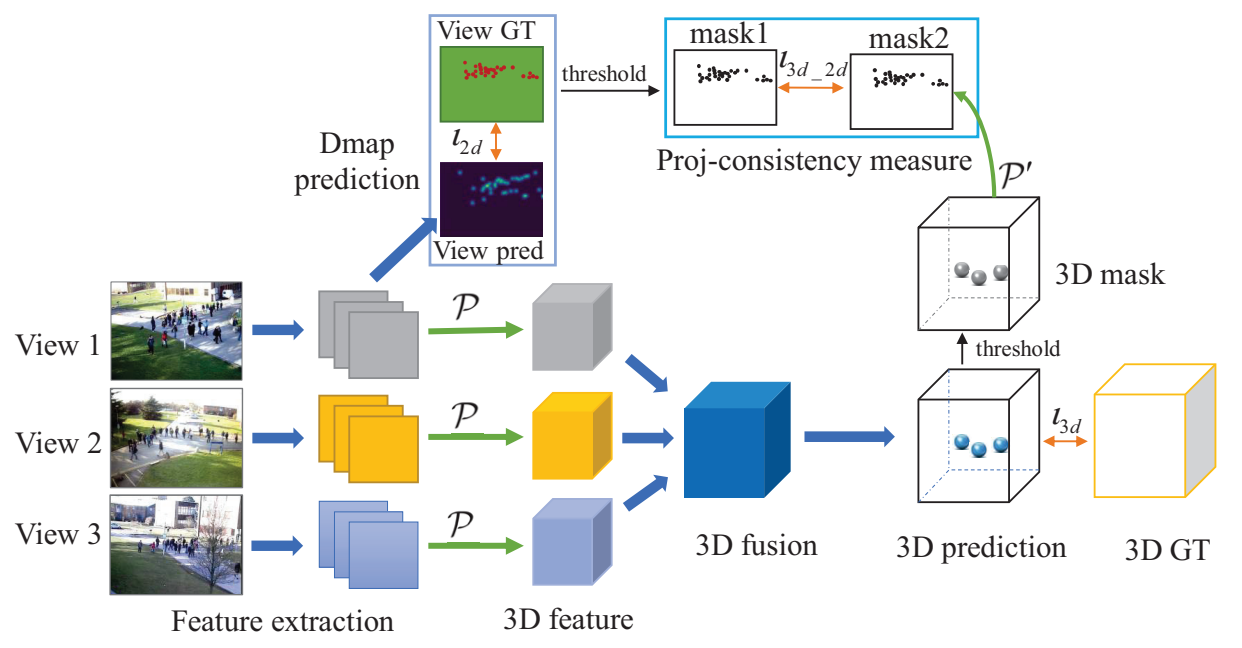

Figure 2: The pipeline of 3D crowd counting. Single-view features are extracted and then projected to the 3D world on multiple height planes. The projected 3D features are concatenated and fused to output the 3D density map prediction (loss $\left.l_{3 d}\right)$. Each camera-view prediction branch decode the $2 \mathrm{D}$ features to obtain the $2 \mathrm{D}$ camera-view predictions (loss $\left.l_{2 d}\right)$. Finally, the $3 \mathrm{D}$ prediction is back-projected to each camera-view, and the the projection consistency between the camera-view ground-truth and the back-projected prediction is measured (loss $\left.l_{3 d_{-} 2 d}\right)$.

The disadvantage of the 2D-to-2D projection in (Zhang and Chan 2019) is that the features of the same person from different views may not line up correctly due to the approximation that all features come from the same height in the $3 \mathrm{D}$ world. Clearly this is not true for features extracted from the heads and feet of the people. To address this problem, in this paper, we propose to use $3 \mathrm{D}$ projection and $3 \mathrm{D}$ feature fusion to perform the multi-view counting task. Our proposed method consists of following components (see Fig. 2): 1) Single view feature extraction and density map prediction: 2D single-view features are extracted and then decoded to the $2 \mathrm{D}$ density map predictions; 2) $2 D$-to-3D projection: A differentiable projection layer together with the camera parameters are used to perform the fixed 3D projection from image plane to 3D world; 3) 3D fusion and prediction: The projected multi-view 3D features are fused using 3D CNN layers to predict the 3D scene-level density map ; 4) Projection consistency between the $3 D$ prediction and $2 D$ views: The 3D prediction is back-projected to each camera view and a loss between the camera view prediction and backprojected 3D prediction is added to enhance the multi-view counting performance.

In our approach, the $3 \mathrm{D}$ projection provides more information of the people, and the projected people's scales along the $z$-dimension in the $3 \mathrm{D}$ world are similar to each other (decided by the people height), which suggests the scale variation issue among multiple views can be tackled without the scale-selection module like in MVMS. In addition, a 3D density map with 3D Gaussian kernels is used to represent the crowd in the scene, instead of the 2D scene-level density maps. The 3D density map can provide more information about the crowd in 3D, e.g., the elevation of the crowd.

In summary, the main contributions of our paper are:

- We propose an end-to-end DNNs-based 3D multi-view counting method that predicts 3D density maps, which provides information about the crowd in 3D. To the best of our knowledge, this is the first study of 3D scene-level multi-view crowd counting.

- Unlike the previous methods, we use 3D projection and $3 \mathrm{D}$ fusion, which can help to deal with the scale variation issue without the scale selection module.

- The projection consistency between the camera-view ground-truth and the back-projected 3D predictions is explored to further boost the multi-view counting performance. The proposed method can achieve better or comparable counting performance to the state-of-art.

\section{Related Work}

In this section, we will review the existing single-view and multi-view counting methods. We also review 3D object reconstruction using deep neural networks (DNNs).

\subsection{Single-view counting}

Single-view crowd counting has achieved satisfactory performance on the existing counting datasets, especially the DNN-based density map estimation methods. Zhang, Li, and et al. 2015 proposed a single-column $\mathrm{CNN}$ framework to directly estimate the density maps from the images. Scale variations due to perspective changes is a critical issue in the crowd counting task, which can limit the performance, and many methods have been proposed to handle scale variations (Boominathan, Kruthiventi, and Babu 2016; Zhang and et al. 2016; Sam, Surya, and Babu 2017; Kang and Chan 2018; Onoro-Rubio and López-Sastre 2016). Sindagi and Patel 2017 proposed the contextual pyramid CNN (CP-CNN) to incorporate global and local context information in the crowd counting framework. Furthermore, extra information and more sophisticated networks were utilized to further 
improve the counting performance (Idrees and et al. 2018; Wang, Gao, and et al. 2019; Ranjan, Le, and Hoai 2018; Cao, Wang, and et al. 2018; Li, Zhang, and Chen 2018; Liu et al. 2018; Shen et al. 2018; Jiang and et al. 2019; Liu, Salzmann, and Fua 2019). Kang, Dhar, and Chan 2017 proposed an adaptive convolution neural network (ACNN) by utilizing the context (camera height and angle) as side information in the counting framework. Shi, Yang, and et al. 2019 integrated the perspective information to provide additional knowledge of the person scale change in an image. Lian et al. 2019 proposed a regression guided detection network (RDNet) for RGB-D crowd counting. Liu and et al. 2019 proposed Recurrent Attentive Zooming Network to zoom high density regions for higher-precision counting and localization.

The single-view based counting methods cannot handle the situations when the camera cannot cover the whole scene, the occlusions in the scene are too severe, or the people are in low resolution due to long distance to the camera. Therefore, multiple cameras should be adopted to improve the counting performance for these wide-area scenes.

\subsection{Multi-view counting}

Traditional multi-view counting methods can be divided into 3 categories: detection/tracking based (Dittrich et al. 2017; Li, Huang, and Liu 2012; Ma, Zeng, and Ling 2012; Maddalena, Petrosino, and Russo 2014), regression based (Ryan et al. 2014; Tang et al. 2014), 3D cylinder based (Ge and Collins 2010). These multi-view counting methods have several limitations. First, they need to utilize foreground extraction techniques to segment the crowd from background. Therefore, the effectiveness of the foreground extraction step limits the final counting performance. Second, hand-crafted features are used both in the people detection or crowd count regression. The hand-crafted features lack representation ability, which reduces the robustness and the performance of the methods. Third, these methods are mainly performed and tested on the PETS2009 (Ferryman and Shahrokni 2009), which is a multi-view dataset with small crowd numbers and staged crowd behavior.

Recently, Zhang and Chan 2019 proposed a DNN-based multi-view counting method MVMS and a new larger multi-view counting dataset CityStreet. MVMS first extracts camera-view information (density maps or features), and then projects them to the average-height plane in the $3 \mathrm{D}$ scene with the given camera parameters. Next, the projected features are fused and decoded to predict the scene-level density maps (on the average height plane). Our proposed method differs from MVMS in the several aspects: 1) instead of using average-height projection, we use multi-height projection, which spatially aligns the person's features (e.g., head, body and feet features) in 3D, making it easier to find the geometric correspondence across views (See Fig. $3)$; 2) we predict a 3D crowd density map, generated using 3D Gaussian kernels, which provides distribution of the crowd in 3D space; 3 ) the 3D density map prediction is backprojected to each camera view, and compared with the $2 \mathrm{D}$ ground-truth density map of the camera view, which defines a projection consistency loss for improving the accuracy.

\subsection{DNN-based 3D reconstruction}

Our 3D crowd counting method is related to a few works on DNN-based 3D object reconstruction and human pose estimation. Yan, Yang, and et al. 2016 proposed an unsupervised single-view 3D object reconstruction method utilizing the projection loss defined by the perspective transformation. Choy et al. 2016 proposed a 3D RNNs architecture to gradually refine the multi-view 3D object reconstruction stepby-step. Kar, Häne, and Malik 2017 leveraged the 3D geometry constraint of features through projection and unprojection operations in the 3D reconstruction method. Huang, Matzen, and et al. 2018 presented DeepMVS to produce a set of plane-sweep volumes and then predict high-quality disparity maps. Iskakov et al. 2019 proposed two learnable triangulation methods for 3D human pose estimation: algebraic triangulation and volumetric aggregation.

It can be observed that the existing DNNs-based 3D reconstruction methods are mainly focused on the reconstruction of a single object (see datasets ShapeNet (Chang and et al. 2015) and IKEA (Lim, Pirsiavash, and Torralba 2013), or human pose). Our proposed method can also be regarded as predicting a 3D representation from multiple viewpoints, but differs from the existing DNN-based 3D object reconstruction in several aspects. First, the proposed method can do more than 3D shape reconstruction, because the 3D Gaussian kernels can represent the crowd's 3D distribution as well as indicate the crowd count. Second, unlike the previous $3 \mathrm{D}$ object reconstruction, the proposed method targets at the scene-level representation (all people in the scene), not only a single object. Furthermore, we exploit the relationship between the 2D camera-view density maps and 3D scene-level density maps to obtain a projection consistency loss.

\section{3D Counting via 3D Projection and Fusion}

We follow the setup of multi-view counting in Zhang and Chan 2019: we assume fixed cameras with known intrinsic and extrinsic camera parameters, and synchronized camera frames across views. In contrast to Zhang and Chan 2019, which is based on 2D ground-plane density maps, we generate 3D ground-truth density maps by convolving the 3D ground-truth annotations with fixed-width 3D Gaussian kernels. The 3D ground-truth annotation coordinates are calculated from the 2D view annotations and people correspondence across views (see Section 4 for more details).

In this section, we introduce the end-to-end DNNs-based 3D crowd counting method consisting of following stages. 1) Single view feature extraction and density map prediction: 2D single-view features are extracted and decoded to predict a 2D camera-view density map; 2) $2 D$-to-3D projection: a differentiable projection layer using the camera parameters projects the camera-view feature maps from the image plane to multiple height-planes in the 3D world; 3) 3D fusion and prediction: the projected multi-view 3D features are fused to predict the 3D density maps using 3D CNN layers; 4) Projection consistency between the $3 D$ prediction and $2 D$ views: the $3 \mathrm{D}$ prediction is back-projected to each camera view, and then compared with the corresponding camera- 


\begin{tabular}{|c|c|c|c|}
\hline \multicolumn{2}{|c|}{ Single-view branch } & & \\
\hline Layer & Filter & & \\
\hline \multicolumn{4}{|c|}{ Feature extraction } \\
\hline \multirow{2}{*}{$\begin{array}{l}\text { conv } 1 \\
\text { conv } 2\end{array}$} & \multirow{2}{*}{$16 \times 1 \times 5 \times 5$} & \multicolumn{2}{|c|}{ 3D Fusion module } \\
\hline & & Layer & Filter \\
\hline pooling & $2 \times 2$ & $\begin{array}{l}\text { concatenation } \\
\text { 3D conv } 1\end{array}$ & $32 \times n \times 5 \times 5 \times 7$ \\
\hline conv 3 & $32 \times 16 \times 5 \times 5$ & $3 \mathrm{D}$ conv 2 & $64 \times 32 \times 5 \times 5 \times 7$ \\
\hline conv 4 & $32 \times 32 \times 5 \times 5$ & 3D conv 3 & $128 \times 64 \times 5 \times 5 \times 7$ \\
\hline pooling & $2 \times 2$ & $\begin{array}{l}3 \mathrm{D} \text { conv } 4 \\
3 \mathrm{D} \text { conv } 5\end{array}$ & $\begin{array}{c}64 \times 128 \times 5 \times 5 \times 7 \\
32 \times 64 \times 5 \times 5 \times 7\end{array}$ \\
\hline Density & nap prediction & 3D conv 6 & $32 \times 32 \times 5 \times 5 \times 7$ \\
\hline conv 5 & $64 \times 32 \times 5 \times 5$ & 3D conv 1 & \\
\hline conv 6 & $32 \times 64 \times 5 \times 5$ & & \\
\hline conv 7 & $1 \times 32 \times 5 \times 5$ & & \\
\hline
\end{tabular}

Table 1: The layer settings for the camera-view feature extraction and density map prediction branches (left), and the 3D fusion module (right). The filter dimensions are output channels, input channels, and filter size $w_{0} \times h_{0} \times d_{0}\left(d_{0}=1\right.$ in 2D conv layers).

view 2D ground-truth using loss to refine the 3D prediction.

\subsection{Single-view branch}

Each camera-view image is fed into a CNN to extract the camera-view features. The 2D camera-view features are decoded by another CNN to predict the camera-view density maps. The camera-view feature extraction and density map prediction layer settings can be found in Table 1 left.

Here, the camera-view prediction branches contribute to the model performance in 2 aspects. First, the camera-view prediction branches together with the $2 \mathrm{D}$ density map supervision improves the training of the camera-view feature extraction. A similar strategy is used in Zhang and Chan 2019, where the camera-view prediction branches are used in the first stage for pre-training. Second, the camera-view prediction branches force the 2D feature representation to be consistent with the 3D feature representation, and the difference between them is the geometric projection. This constraint is natural because the $2 \mathrm{D}$ and $3 \mathrm{D}$ observations are linked by the geometry projection, and the same link still exists between the $2 \mathrm{D}$ and $3 \mathrm{D}$ feature representations. The 2D-3D feature representation constraint was also used in Girdhar et al. 2016, where the $3 \mathrm{D}$ representation is forced to be able to predict the 2D observations from it. Furthermore, this constraint can improve the final 3D prediction performance (see ablation study for more details).

Suppose there are $N$ camera-views, the density map prediction for the $i_{t h}$ view is $V_{i} \in R^{h \times w}$ and the corresponding ground-truth is $V_{i}^{t} \in R^{h \times w}$, then the camera-view density map prediction loss $l_{2 d}$ is the mean-square error (MSE):

$$
l_{2 d}=\frac{1}{w h} \sum_{i=1}^{N}\left\|V_{i}-V_{i}^{t}\right\|_{2}^{2} .
$$

\subsection{D-to-3D projection}

The extracted camera-view features are projected to the 3D world with a fixed 2D-to-3D projection layer. We assume that the camera intrinsic and extrinsic parameters are both

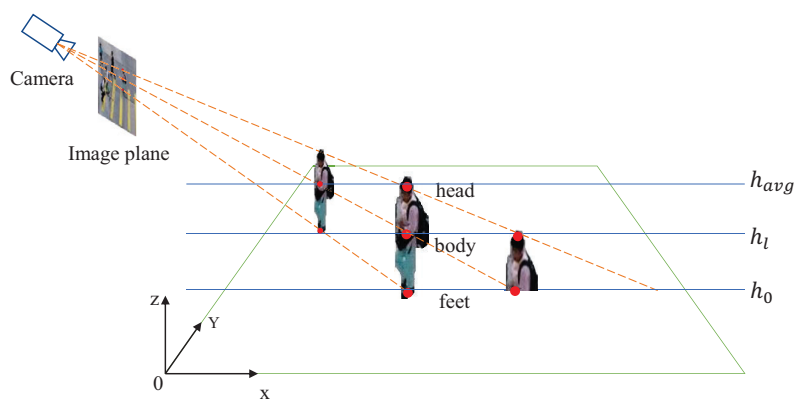

Figure 3: The multi-height projection can extract feature of the people body along $z$ dimension and form a 3D feature representation for the people, which is consistent with the 3D scene.

known, which are used to perform the projection. Since each image pixel's corresponding height in the 3D world is unknown, a height range $H$ is used in the projection, where each pixel is projected to the 3D world multiple times onto different height planes. Then, the projected features from all height are concatenated along the $z$-dimension to form a 3D feature representation. The 2D-to-3D projection layer can be implemented using the Sampler from the Spatial Transformer Networks (Jaderberg et al. 2015) with the known camera calibration parameters (intrinsic and extrinsic). Suppose that $\mathcal{P}$ is the $2 \mathrm{D}$-to-3D projection, $F_{i}$ stands for the 2D feature presentation of view $i$, and the height range $H=\left\{h_{0}, h_{1}, \ldots, h_{r}\right\}$, then the projected 3D feature representation $\left(F_{3 d}\right)_{i}$ for view $i$ is

$$
\left(F_{3 d}\right)_{i}=\mathcal{P}_{i}\left(F_{i}, H\right)=\left[\mathcal{P}_{i}\left(F_{i}, h_{0}\right), \ldots, \mathcal{P}_{i}\left(F_{i}, h_{r}\right)\right],
$$

where $[\cdot]$ is concatenation along the $z$-dimension (height).

In Zhang and Chan 2019, the fixed-height projection was used where all pixels were projected to the average height $(1750 \mathrm{~mm})$. Compared to average-height projection, the multi-height projection can output a body feature representation along $z$-dim (e.g., head, body and feet features, see Fig. 3), which allows the network to better identify the location of the person through the $3 \mathrm{D}$ alignment of the features.

\subsection{D density map prediction}

After the 2D-to-3D projection, the projected 3D features from multiple views are concatenated (along the feature channel) and then decoded by several 3D convolution layers. The architecture for the 3D feature decoder layers can be found in Table 1 right. Suppose the 3D prediction is $G \in R^{a \times b \times d}$ and the corresponding ground-truth is $G^{t} \in$ $R^{a \times b \times d}$, the $3 \mathrm{D}$ prediction loss $l_{3 d}$ is the MSE

$$
l_{3 d}=\frac{1}{a b d}\left\|G-G^{t}\right\|_{2}^{2} .
$$

\subsection{D-to-2D projection consistency measure}

Considering the geometry constraint between 2D observations and the $3 \mathrm{D}$ observation, we also require the $3 \mathrm{D}$ prediction be consistent with the $2 \mathrm{D}$ single view density maps in 


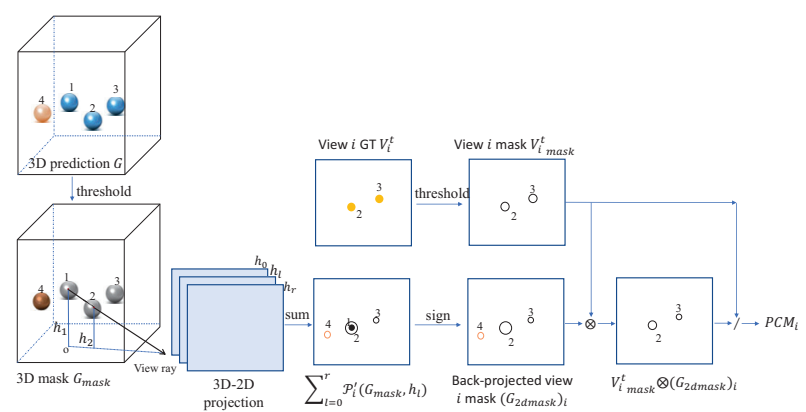

Figure 4: Example of projection consistency measure (PCM). There are 4 people in the 3D prediction, while only Person 2 and Person 3 are visible in the 2D view $i$. Since Person 1 is occluded by Person 2 and Person 4 is totally occluded in view $i$, they are masked out in the PCM calculation. When the people location in the $3 \mathrm{D}$ prediction is not consistent with the $2 \mathrm{D}$ ground-truth, the $P C M$ value is low.

terms of projection geometry. To achieve this, the 3D prediction $G$ is projected to each camera-view $i$ with a 3Dto-2D projection operation $\mathcal{P}^{\prime}$. The projection consistency between the projected $2 \mathrm{D}$ density maps and the $2 \mathrm{D}$ density map ground-truth is measured and used as part of the loss to further enhance the 3D counting performance.

3D-to-2D projection. Suppose the $\mathcal{P}^{\prime}$ is the 3D-to-2D projection, $G_{\text {mask }}$ is a 3D binary mask which is the 3D prediction $G$ thresholded at $T=10^{-4}$. When performing the projection, each pixel's height of the $2 \mathrm{D}$ view plane is also unknown (each 2D image point is corresponding to many $3 \mathrm{D}$ points along the view ray without the height information). Therefore, the height range $H=\left\{h_{0}, h_{1}, \ldots, h_{r}\right\}$ is also used in the 3D-to-2D projection. Then the projected $2 \mathrm{D}$ mask $\left(G_{2 d m a s k}\right)_{i}$ for view $i$ can be denoted as,

$$
\left(G_{2 d m a s k}\right)_{i}=\operatorname{sign}\left(\sum_{l=0}^{r} \mathcal{P}_{i}^{\prime}\left(G_{m a s k}, h_{l}\right)\right),
$$

where $\sum$ is summation along the $z$-dimension (height dimension). In the $3 \mathrm{D}$-to-2D projection, the projected $2 \mathrm{D}$ pixel value can be regarded as the binary summation along the view ray in the 3D prediction grid (see Fig. 4).

Projection consistency measure. The main challenge to measure the consistency between the 3D density map prediction and the 2D camera-view density map is that some people who are occluded in a $2 \mathrm{D}$ camera-view will be present in the 3D prediction (because they are visible from other views). Thus the MSE loss cannot be used directly here. Instead, we measure the consistency between the binary masks produced by thresholding the 3D prediction and the corresponding 2D ground-truth density map, while accounting for inconsistencies due to occlusions (see Fig. 4).

We define the 3D-to-2D projection consistency measure (PCM) for view $i$ :

$$
P C M_{i}=\frac{\left\|V_{i \text { mask }}^{t} \otimes\left(G_{2 \text { dmask }}\right)_{i}\right\|_{1}}{\left\|V_{i \text { mask }}^{t}\right\|_{1}+\alpha},
$$

where $\otimes$ denotes element-wise multiplication, $V_{i \text { mask }}^{t}$ is a binary mask which is the ground-truth density map of view $i$ thresholded at $1 \mathrm{e}-3,\left(G_{2 d \text { mask }}\right)_{i}$ is the back-projected 3D prediction mask, and $\alpha=1 \mathrm{e}-5$ is a constant to prevent divide-by-zero. The important property of the PCM is that no penalty occurs when an extra person is present in the 3D prediction $\left(\left(G_{2 d m a s k}\right) i\right)$, but not the 2D camera-view (e.g., due to occlusion). On the other hand, the PCM will be reduced when a person in the $2 \mathrm{D}$ camera-view is missing in the $3 \mathrm{D}$ prediction. Finally, the projection consistency loss is defined by summing over the camera-views:

$$
l_{3 d_{-} 2 d}=\sum_{i=1}^{N}\left(1-P C M_{i}\right) .
$$

\subsection{Training loss}

Combining all the aforementioned losses, the final loss is

$$
l_{\text {all }}=l_{3 d}+\beta l_{2 d}+\gamma l_{3 d_{-} 2 d},
$$

where $\beta$ and $\gamma$ are hyperparameters for weighting the contributions of each term.

\section{Experiments and Evaluation}

In this section, we will discuss the implementation details, and conduct experiments of our proposed 3D crowd counting framework on three multi-view counting datasets.

\subsection{Implementation details}

3D ground-truth generation. The 3D ground-truth is generated with the single-view annotations (here we use head annotations) and the cross-view correspondence. Suppose the same person's corresponding annotations in $M$ views $(M \leq N)$ are $\left\{\left(x_{j}, y_{j}\right)\right\}, j \in\{1,2, \ldots, M\}$, their corresponding 3D coordinates are denoted as $\left\{W_{j}\right\}=$ $\left\{\mathcal{P}_{j}\left(\left(x_{j}, y_{j}, h\right)\right)\right\}$ where the height $h$ is not known. Considering the projection correspondence, the $3 \mathrm{D}$ coordinate of the person's head $W=[X, Y, Z]^{T}$ can be obtained by finding the height $h$ that minimizes the spread of the projected head positions from all views,

$$
\begin{aligned}
& Z=\underset{h}{\operatorname{argmin}}\left\|W_{j}-\bar{W}\right\|_{2}^{2}, \\
& (X, Y)=\frac{1}{M} \sum_{j=1}^{M} \mathcal{P}_{j}\left(\left(x_{j}, y_{j}, Z\right)\right),
\end{aligned}
$$

where $\bar{W}$ is the mean of $\left\{W_{j}\right\} . Z$ is the height $h$ minimizing (8), which can be obtained through searching $h$ in the height range $H_{0} \in\left\{h_{0}, h_{1}, \ldots, h_{r 0}\right\}$ (we use range $\{1000,1010,1020, \ldots, 2000 \mathrm{~mm}\})$. For numerical stability during training, 2D density ground-truth maps are scaled by $10^{3}$, while the $3 \mathrm{D}$ ground-truth is scaled by $10^{4}$. The predictions are scaled downward correspondingly for evaluation.

Training settings and methods. In the proposed method (see Fig. 2), except the 3D counting prediction, there are two important modules: single-view density map prediction and the projection consistency measure module. In the ablation study, we show the two modules' influence to the final 3D counting performance. A 3-stage training is used for 


\begin{tabular}{l|c|c|c}
\hline Dataset & PETS2009 & DukeMTMC & CityStreet \\
\hline Dmap weighted & 8.32 & 2.12 & 9.36 \\
Detection+ReID & 9.41 & 2.20 & 27.60 \\
\hline Late fusion (Zhang and Chan 2019) & 3.92 & 1.27 & 8.12 \\
Naïve early fusion (Zhang and Chan 2019) & 5.43 & 1.25 & 8.10 \\
MVMS (Zhang and Chan 2019) & 3.49 & $\mathbf{1 . 0 3}$ & 8.01 \\
\hline 3D counting (ours) & $\mathbf{3 . 1 5}$ & 1.37 & $\mathbf{7 . 5 4}$ \\
\hline
\end{tabular}

Table 2: Experiment results: mean absolute error (MAE) on three multi-view counting datasets. "3D counting" uses both singleview branches and the projection consistency measure loss.

\begin{tabular}{l|ccc|ccc|ccc}
\hline Dataset & \multicolumn{3}{|c|}{ PETS2009 } & \multicolumn{3}{c|}{ DukeMTMC } & \multicolumn{3}{c}{ CityStreet } \\
\hline$n * h$ & $3 \mathrm{D}$ & 3D+2D & 3D+2D+PCM & 3D & 3D+2D & 3D+2D+PCM & 3D & 3D+2D & 3D+2D+PCM \\
\hline $7 * 40 \mathrm{~cm}$ & 4.12 & 3.20 & $\mathbf{3 . 1 5}(\gamma=100)$ & 1.82 & 1.71 & $1.65(\gamma=10)$ & 8.98 & 8.49 & $8.35(\gamma=100)$ \\
$14 * 20 \mathrm{~cm}$ & 4.88 & 4.57 & $4.24(\gamma=10)$ & 2.12 & 1.63 & $1.49(\gamma=3)$ & 8.72 & 7.89 & $7.71(\gamma=30)$ \\
$28 * 10 \mathrm{~cm}$ & 5.34 & 4.27 & $4.21(\gamma=1)$ & 2.15 & 1.41 & $\mathbf{1 . 3 7}(\gamma=0.5)$ & 7.87 & 7.58 & $\mathbf{7 . 5 4}(\gamma=10)$ \\
\hline
\end{tabular}

Table 3: Ablation study of the training loss and ground-truth settings. $\gamma$ is the hyperparameter for the projection consistency measure (PCM) loss. $n$ is the number of voxels in the $z$-dimension (height), and $h$ is the voxel height in the 3D world. For DukeMTMC, the number of voxels in $z$-dimension is slightly larger $(9,18,36)$.

the proposed method: In the first stage, $\beta=1$ means that the single-view 2D supervision is dominant to benefit the feature extraction training; In the second stage, $\beta=0.01$ means the $3 \mathrm{D}$ supervision is dominant to accelerate the $3 \mathrm{D}$ fusion training; In the third stage, $\beta=0.01$ and $\gamma$ is variable according to ground-truth setting, which increases the influence of the projection consistency measure (PCM) to further enhance the performance. In all stages, the learning rate is set as $10^{-4}$ and the batch size is 1 .

\subsection{Evaluation methods}

Datasets. The proposed method is evaluated and compared on the 3 multi-view counting datasets, PETS2009 (Ferryman and Shahrokni 2009), DukeMTMC (Ristani, Solera, and et al. 2016) and CityStreet (Zhang and Chan 2019). We directly use the same dataset settings as in Zhang and Chan 2019. PETS2009 contains 3 views, and 1105 and 794 images are for training and testing, respectively. The input image resolution $(w \times h \times d)$ is $384 \times 288$ and the $3 \mathrm{D}$ ground-truth resolution is $152 \times 177 \times 7$. The voxel height in $z$-dim is $40 \mathrm{~cm}$ in the real world and the height range is $0-2.8 \mathrm{~m}$. DukeMTMC (Ristani, Solera, and et al. 2016) contains 4 views, and the first 700 images are used for training and remaining 289 images are for testing. The input image resolution is $640 \times 360$ and the $3 \mathrm{D}$ ground-truth resolution is $160 \times 120 \times 36$. The voxel height in $z$-dim is $10 \mathrm{~cm}$ in the real world and the height range is $0-3.6 \mathrm{~m}$. CityStreet (Zhang and Chan 2019) consists of 3 views and 500 images in which the first 300 for training and rest 200 for testing. The input image resolution is $676 \times 380$ and the $3 \mathrm{D}$ ground-truth resolution is $160 \times 192 \times 28$. The voxel height in $z$-dim is $10 \mathrm{~cm}$ in the real world and the height range is $0-2.8 \mathrm{~m}$.

Comparison methods. 5 multi-view counting methods are used for comparison. 1) "Dmap weighted" fuses singleview density maps into a scene-level count with a viewspecific weighted map, which is constructed based on how many views can see a particular pixel. In the experiment, CSR-net (Li, Zhang, and Chen 2018) is used to predict the single-view density maps for datatsets PETS2009 and CityStreet, and FCN-7 for DukeMTMC. 2) "Detection + ReID" first detects all humans in each camera-view and then the scene geometry constraints and person ReID are used to associate the same people across views. Specifically, Faster-RCNN (Ren et al. 2015) is used for people detection and LOMO 2015 (Liao et al. 2015) for person ReID. 3) "late fusion" model fuses single-view density maps to predict 2D height-plane density maps (Zhang and Chan 2019); 4) "naïve early fusion" model fuses feature maps to predict 2D height-plane density maps (Zhang and Chan 2019); 5) "multi-view multi-scale (MVMS)" model fuses feature maps with a scale selection module to cope with the scale variation issue (Zhang and Chan 2019).

Evaluation metric. The mean absolute error (MAE) is used to evaluate the scene-level counting performance, comparing the scene-level predicted and ground-truth counts.

\subsection{Experiment results}

The experimental results are shown in Table 2 and the visualization results can be found in the Fig. 5 (also see the Supplemental). On PETS2009, the proposed 3D multi-view counting method can achieve better results than the two baseline multi-view counting methods ("Dmap weighted", "Detection + ReID") and the 3 versions of the end-to-end multi-view counting method proposed in Zhang and Chan 2019. The first two baseline methods cannot effectively fuse the multi-view information, which limits their performance. The proposed method achieves better performance than MVMS (Zhang and Chan 2019), which shows the advantage of the 3D projection and 3D fusion. On DukeMTMC, our 3D multi-view counting method achieves comparable performance to the MVMS (Zhang and Chan 2019). But the proposed method still achieves better performance than the two baseline methods. Due to low crowd count and lack of occlusions in the DukeMTMC, the performance gap is not very obvious. On CityStreet, our 3D multi-view counting method achieves the best results among the end-to-end 


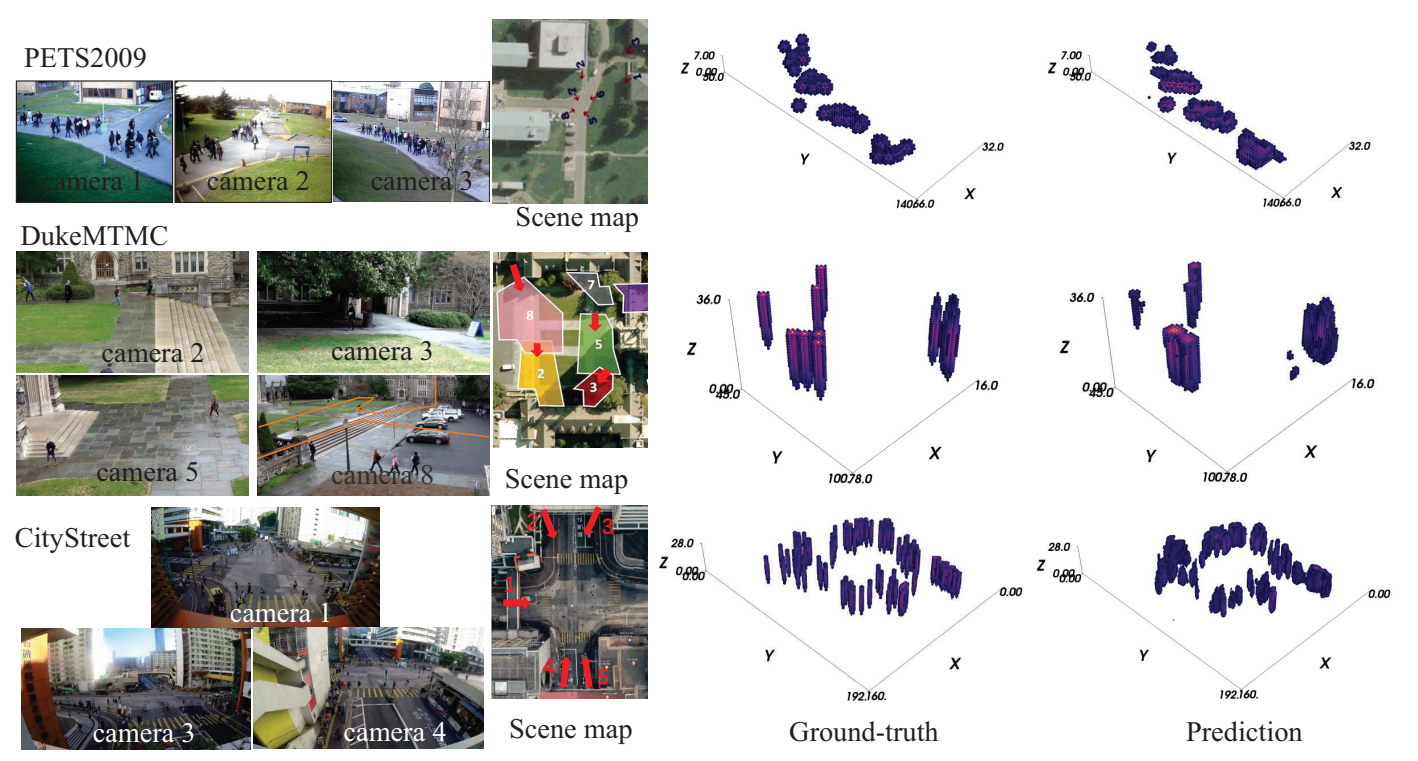

Figure 5: Examples of the 3 multi-view datasets and their prediction results. The 3D density maps of PETS2009, DukeMTMC and CityStreet are thresholded by 5e-3, 1e-3 and 1e-3, respectively. See the supplemental for more visualizations.

multi-view counting method (late fusion, naive early fusion, MVMS) and the two baseline methods. "Detection + ReID" performs badly on CityStreet due to large crowd count and severe occlusions.

\subsection{Ablation study}

In this section, we perform ablation studies on the training loss and the ground-truth settings.

Training loss. The rows of Table 3 show the results of using 3D loss, 3D+2D loss or $3 \mathrm{D}+2 \mathrm{D}+\mathrm{PCM}$ loss on the 3 datasets. It can be observed using single-view prediction branches and 2D supervision (3D+2D loss) can achieve better multi-view counting performance in comparison with only using $3 \mathrm{D}$ loss. Furthermore, using $3 \mathrm{D}+2 \mathrm{D}$ together with PCM loss can obtain better multi-view counting performance compared to using only $3 \mathrm{D}$ loss or $3 \mathrm{D}+2 \mathrm{D}$ loss on all 3 datasets with different ground-truth settings.

Ground-truth setting. The columns of Table 3 show the results of using different resolution of the 3D density map ( $n$ is the number of voxels in the $z$-dimension, and $h$ is the voxel height in the 3D world). For PETS2009, the best performance is achieved by using voxel number 7 and voxel height $40 \mathrm{~cm}$ with $3 \mathrm{D}+2 \mathrm{D}+\mathrm{PCM}$ loss $(\gamma=100)$. For DukeMTMC, using voxel number 36 and voxel height $10 \mathrm{~cm}$ with $3 \mathrm{D}+2 \mathrm{D}+\mathrm{PCM} \operatorname{loss}(\gamma=0.5)$ gives the best result. As to CityStreet, the best result is obtained by using voxel number 28 and voxel height $10 \mathrm{~cm}$ with $3 \mathrm{D}+2 \mathrm{D}+\mathrm{PCM}$ loss $(\gamma=10)$. Compared to DukeMTMC and CityStreet, the best performance of PETS2009 is achieved at $h=40 \mathrm{~cm}$. The people occlusion in PETS2009 is more severe and many people's lower bodies are totally occluded from all views (e.g., the people in the middle). Thus, increasing the height resolution does not provide additional information of the body, but may introduce more noises (other people's features) along the $z$-dim, thus leading to worse performance.

\section{Conclusion and Discussion}

In this paper, a DNN-based 3D multi-view counting method is proposed, which fuses camera-views to predict the 3D scene-level density map. 3D projection and fusion are used, which can handle the situation when people are not all located at the same height (e.g., people standing on a staircase), and provides a chance to solve the scale variation issue in the 3D space without a scale selection operation. The projection consistency measure between the 3D prediction and 2D density map ground-truth is studied and then utilized in the loss function to refine the 3D prediction further. Compared to other state-of-art multi-view counting methods, the proposed method achieves better or comparable counting performance as well as a more informative scenelevel crowd representation.

In addition to counting humans, the proposed 3D multiview counting method can also be applied to counting birds in the sky or the fish in the aquarium, where both the bird or the fish count can be obtained as well as their 3D location distributions - of course, this requires collecting more multi-view scenes. Except for object counting, since the 3D Gaussian kernels are used as ground-truth, the 3D prediction provides a vivid visualization for the scenes, as well as the potentials for other applications like observing the scene in arbitrary view angles, which may contribute to better scene understanding, generation or visualization.

\section{Acknowledgements}

This work was supported by grants from the Research Grants Council of the Hong Kong Special Administrative Region, China (Project No. [T32-101/15-R] and CityU 11212518). 


\section{References}

Boominathan, L.; Kruthiventi, S. S.; and Babu, R. V. 2016. Crowdnet: A deep convolutional network for dense crowd counting. In ACM Multimedia Conference, 640-644. ACM.

Cao, X.; Wang, Z.; and et al. 2018. Scale aggregation network for accurate and efficient crowd counting. In ECCV, 734-750.

Chan, A. B.; Liang, Z. S. J.; and Vasconcelos, N. 2008. Privacy preserving crowd monitoring: Counting people without people models or tracking. In $C V P R, 1-7$.

Chang, A. X., and et al. 2015. Shapenet: An information-rich 3d model repository. arXiv preprint arXiv:1512.03012.

Chen, K.; Chen, L. C.; Gong, S.; and Xiang, T. 2012. Feature mining for localised crowd counting. In $B M V C$.

Choy, C. B.; Xu, D.; Gwak, J.; Chen, K.; and Savarese, S. 2016. 3d-r2n2: A unified approach for single and multi-view $3 \mathrm{~d}$ object reconstruction. In ECCV, 628-644. Springer.

Dittrich, F.; de Oliveira, L. E.; Britto Jr, A. S.; and Koerich, A. L. 2017. People counting in crowded and outdoor scenes using a hybrid multi-camera approach. arXiv preprint arXiv:1704.00326.

Ferryman, J., and Shahrokni, A. 2009. Pets2009: Dataset and challenge. In IEEE International Workshop on Performance Evaluation of Tracking and Surveillance, 1-6.

Ge, W., and Collins, R. T. 2010. Crowd detection with a multiview sampler. In ECCV, 324-337.

Girdhar, R.; Fouhey, D. F.; Rodriguez, M.; and Gupta, A. 2016. Learning a predictable and generative vector representation for objects. In $E C C V, 484-499$. Springer.

Huang, P.-H.; Matzen, K.; and et al. 2018. Deepmvs: Learning multi-view stereopsis. In CVPR, 2821-2830.

Idrees, H., and et al. 2018. Composition loss for counting, density map estimation and localization in dense crowds. In ECCV, 532546.

Idrees, H.; Saleemi, I.; Seibert, C.; and Shah, M. 2013. Multisource multi-scale counting in extremely dense crowd images. In CVPR, 2547-2554.

Iskakov, K.; Burkov, E.; Lempitsky, V.; and Malkov, Y. 2019. Learnable triangulation of human pose. In $I C C V$.

Jaderberg, M.; Simonyan, K.; Zisserman, A.; and Kavukcuoglu, K. 2015. Spatial transformer networks. In Advances in Neural Information Processing Systems, 2017-2025.

Jiang, X., and et al. 2019. Crowd counting and density estimation by trellis encoder-decoder networks. In CVPR, 6133-6142.

Kang, D., and Chan, A. 2018. Crowd counting by adaptively fusing predictions from an image pyramid. In $B M V C$.

Kang, D.; Dhar, D.; and Chan, A. 2017. Incorporating side information by adaptive convolution. In Advances in Neural Information Processing Systems, 3867-3877.

Kar, A.; Häne, C.; and Malik, J. 2017. Learning a multi-view stereo machine. In NIPS, 365-376.

Li, J.; Huang, L.; and Liu, C. 2012. People counting across multiple cameras for intelligent video surveillance. In IEEE Ninth International Conference on Advanced Video and Signal-Based Surveillance (AVSS), 178-183. IEEE.

Li, Y.; Zhang, X.; and Chen, D. 2018. Csrnet: Dilated convolutional neural networks for understanding the highly congested scenes. In CVPR, 1091-1100.

Lian, D.; Li, J.; Zheng, J.; Luo, W.; and Gao, S. 2019. Density map regression guided detection network for rgb-d crowd counting and localization. In $C V P R, 1821-1830$.
Liao, S.; Hu, Y.; Zhu, X.; and Li, S. Z. 2015. Person reidentification by local maximal occurrence representation and metric learning. In $C V P R, 2197-2206$.

Lim, J. J.; Pirsiavash, H.; and Torralba, A. 2013. Parsing IKEA Objects: Fine Pose Estimation. In ICCV.

Liu, C., and et al. 2019. Recurrent attentive zooming for joint crowd counting and precise localization. In $C V P R, 1217-1226$.

Liu, J.; Gao, C.; Meng, D.; and Hauptmann, A. G. 2018. Decidenet: Counting varying density crowds through attention guided detection and density estimation. In CVPR, 5197-5206.

Liu, W.; Salzmann, M.; and Fua, P. 2019. Context-aware crowd counting. In CVPR, 5099-5108.

Ma, H.; Zeng, C.; and Ling, C. X. 2012. A reliable people counting system via multiple cameras. ACM Transactions on Intelligent Systems and Technology (TIST) 3(2):31.

Maddalena, L.; Petrosino, A.; and Russo, F. 2014. People counting by learning their appearance in a multi-view camera environment. Pattern Recognition Letters 36:125-134.

Onoro-Rubio, D., and López-Sastre, R. J. 2016. Towards perspective-free object counting with deep learning. In $E C C V$, 615-629. Springer.

Ranjan, V.; Le, H.; and Hoai, M. 2018. Iterative crowd counting. In ECCV, 270-285.

Ren, S.; He, K.; Girshick, R.; and Sun, J. 2015. Faster r-cnn: Towards real-time object detection with region proposal networks. In Advances in neural information processing systems, 91-99.

Ristani, E.; Solera, F.; and et al. 2016. Performance measures and a data set for multi-target, multi-camera tracking. In ECCV workshop on Benchmarking Multi-Target Tracking.

Ryan, D.; Denman, S.; Fookes, C.; and Sridharan, S. 2014. Scene invariant multi camera crowd counting. Pattern Recognition Letters 44(8):98-112.

Sam, D. B.; Surya, S.; and Babu, R. V. 2017. Switching convolutional neural network for crowd counting. In CVPR, 4031-4039.

Shen, Z.; Xu, Y.; Ni, B.; Wang, M.; Hu, J.; and Yang, X. 2018. Crowd counting via adversarial cross-scale consistency pursuit. In CVPR, 5245-5254.

Shi, M.; Yang, Z.; and et al. 2019. Revisiting perspective information for efficient crowd counting. In CVPR, 7279-7288.

Sindagi, V. A., and Patel, V. M. 2017. Generating high-quality crowd density maps using contextual pyramid cnns. In ICCV, $1879-1888$.

Tang, N.; Lin, Y. Y.; Weng, M. F.; and Liao, H. Y. 2014. Crosscamera knowledge transfer for multiview people counting. IEEE Transactions on Image Processing 24(1):80-93.

Wang, Q.; Gao, J.; and et al. 2019. Learning from synthetic data for crowd counting in the wild. In CVPR, 8198-8207.

Yan, X.; Yang, J.; and et al. 2016. Perspective transformer nets: Learning single-view $3 \mathrm{~d}$ object reconstruction without $3 \mathrm{~d}$ supervision. In NIPS, 1696-1704.

Zhang, Q., and Chan, A. B. 2019. Wide-area crowd counting via ground-plane density maps and multi-view fusion cnns. In $C V P R$, 8297-8306.

Zhang, Y., and et al. 2016. Single-image crowd counting via multicolumn convolutional neural network. In CVPR, 589-597.

Zhang, C.; Li, H.; and et al. 2015. Cross-scene crowd counting via deep convolutional neural networks. In CVPR, 833-841. 